\title{
IDENTIFICACIÓN Y CLASIFICACIÓN DE SUSTANCIAS QUÍMICAS CANCERÍGENAS EN UNA PLANTA PROCESADORA DE ALIMENTOS CÁRNICOS
}

\author{
IDENTIFICATION AND CLASSIFICATION OF CARCINOGENIC \\ CHEMICAL SUBSTANCES IN A MEAT PROCESSING PLANT
}

\section{${ }^{1}$ Angela Daniella Arias Daza, ${ }^{2}$ Efren Venancio Ramos Cabrera, ${ }^{3}$ Zuly Yuliana Delgado Espinosa}

\author{
${ }^{1}$ Ingeniera Industrial, Corporación universitaria comfacauca-Uniconfacauca. Popayán - Colombia. \\ ${ }^{2}$ Ph. D. Microbiología, Corporación Universitaria Comfacauca-Unicomfacauc. Popayán, Colombia. \\ ${ }^{3}$ PhD en Ciencias Química, Corporación Universitaria Comfacauca-Unicomfacauc. Popayán, Colombia.
}

${ }^{1}$ anagelaarias@unicomfacauca.edu.co, ${ }^{2}$ eramos@unicomfacauca.edu.co, ${ }^{3}$ zdelgado@unicomfacauca.edu.co

Citación: Arias, A., Ramos, E., y Delgado, Z. (2021). Identificación y clasificación de sustancias químicas cancerígenas en una planta procesadora de alimentos cárnicos. Revista de Investigación Agraria y Ambiental, $12(2), 147$ - 160. DOI: https://doi.org/10.22490/21456453.3881

\section{RESUMEN}

Contextualización: en la industria y sectores tecnológicos se utiliza una gran cantidad de productos químicos, generando riesgos para la salud de quienes están expuestos. Esto puede inducir alteraciones dependiendo de la concentración, manipulación, exposición, susceptibilidad del trabajador. Estos agentes químicos pueden producir una diversidad de efectos irritantes alérgicos, tóxicos e incluso cancerígenos.

Vacío de investigación: el desconocimiento de la peligrosidad sumado a las condiciones de operación de sustancias químicas cancerígenas que se presentan en el ejercicio de las actividades diarias en la industria puede afectar la salud Humana.

Propósito: el objetivo del estudio es identificar y clasificar el riesgo relacionado con esas sustancias químicas en una planta procesadora de alimentos cárnicos en el departamento del Cauca (Colombia).

Metodología: en una primera fase de diagnóstico se realizó una búsqueda de información en bases de datos especializadas. La segunda fase se desarrolla a partir de la información recolectada mediante el inventario de sustancias químicas en la planta, que se encuentran distribuidas en diferentes sitios de almacenamiento en los que existe una probabilidad de exposición. Para identificar la estimación del riesgo químico se aplicó un análisis cualitativo mediante la utilización del criterio de caracterización de riesgos "caja de herramientas de control químico internacional". Posteriormente se hizo una revisión de normatividad que rige en Colombia relacionada a la manipulación, 
aplicativos y requisitos correspondientes de estas sustancias, estableciendo medidas de control y prevención necesarias a la naturaleza química de estos agentes.

Resultados y conclusiones: mediante el inventario, se identificaron 407 sustancias presentes en la planta, de las cuales un $6 \%$ no representan clasificación peligrosa, el $91 \%$ se clasifican dentro de sustancias químicas peligrosas y un $3 \%$ corresponde a sustancias químicas con potencial efecto cancerígeno por sus propiedades toxicológicas y efectos específicos sobre la salud humana. Mediante la evaluación de riesgo se tuvo en cuenta la combinación de factores de peligro para la salud y exposición potencial que determinan el nivel de control deseado, dividida en cuatro niveles de abordaje $(1,2,3,4)$ siendo el abordaje 4 de consideración especial por ser el punto más crítico y de mayor peligrosidad. Finalmente, se determinó que las sustancias de mayor atención en la planta son: el cemento portland, DQO solución B, Gasolina, Sikadur 32 Primer Comp B, Sikadur Panel Comp B, por estar dentro de abordaje 4.

Palabras clave: riesgo, peligro de exposición, evaluación del riesgo químico, sustancias químicas peligrosas, sustancias cancerígenas

\section{ABSTRACT}

Contextualization: A large number of chemical products are used in industry and technological sectors, generating health risks for those who are exposed, and may induce alterations depending on the concentration, manipulation, exposure, and susceptibility of the worker. These chemical agents can produce a variety of irritating allergic, toxic and even carcinogenic effects.

Knowledge gap: Lack of knowledge of the danger, together with the operating conditions of chemical carcinogenic chemicals that occur in the exercise of daily activities in the industry may affect human health.

Purpose: The objective of the study is to identify and classify the risk related to these chemical substances in a meat food processing plant in the department of Cauca (Colombia).

Methodology: In the first stage of diagnosis, an information search was carried out in specialized databases; in the second stage it was developed from the information collected through the inventory of chemical substances in the plant, which are distributed at different storage sites where there is probability of exposure. To identify the chemical risk identification, a qualitative analysis is applied using the risk characterization criterion "international chemical control toolbox". Subsequently, a review of the regulations in force in Colombia related to the handling, applications and corresponding requirements of these substances was made, establishing necessary control and prevention measures for the chemical nature of these agents.

Results and conclusions: Through the inventory, 407 substances present in the plant were identified, $91 \%$ of which are classified as dangerous chemicals and 3\% corresponds to chemicals with a potential carcinogenic effect. The risk assessment took into account the combination of risk factors for health and potential exposure that determines the level of control desired, divided into four levels of approach $(1,2,3,4)$, with approach 4 being considered Special for being the most critical and most dangerous point. Finally, it was determined that the substances of greatest attention in the plant are: portland cement, COD solution B, Gasoline, Sikadur 32 Primer Comp B, Sikadur Panel Comp B, for being within the approach 4.

Keywords: Risk, exposure hazard, chemical risk assessment, dangerous chemical substances, carcinogenic substances 


\section{RESUMEN GRÁFICO}

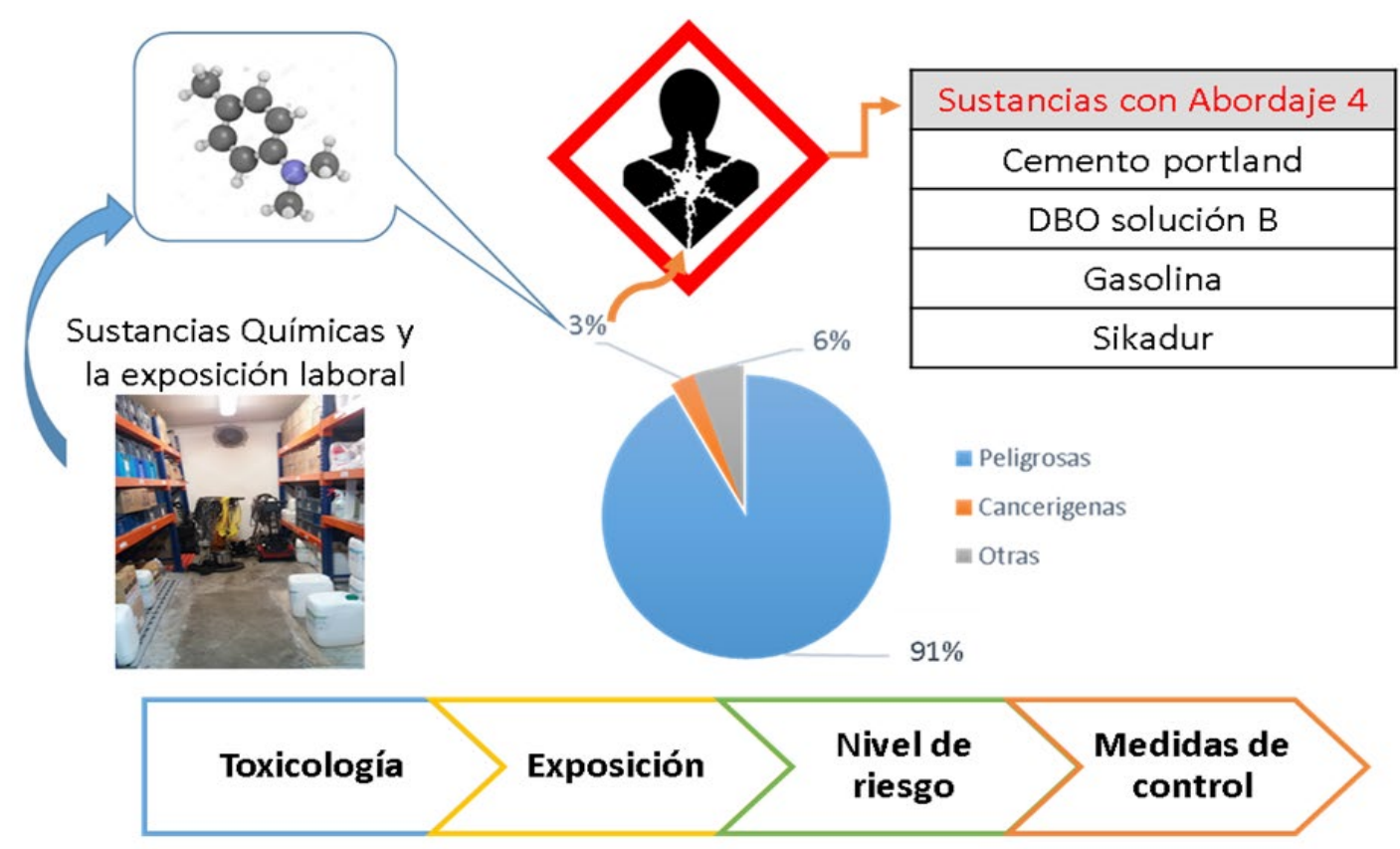

Fuente: Autores

\section{INTRODUCCIÓN}

En el mundo, las sustancias químicas se utilizan en casi todos los sectores productivos, siendo aplicadas regularmente en procesos industriales. Un gran porcentaje de estas sustancias es considera como peligrosas, debido a que algunas de sus características o propiedades afectan al ambiente y la salud humana. Se han identificado más de 25 millones de sustancias químicas peligrosas, de las cuales se comercializan y están registradas 100.195 en la Unión Europea (Sistema globalmente armonizado de Clasificación y etiquetado de productos químicos, 2013). Las empresas han usado hasta 30.000 de estos productos, de los que el $66 \%$ no se les ha realizado pruebas toxicológicas y el $21 \%$ de las sustancias de mayor demanda en la producción carecen de información toxicológica (Calera y Alonso, 2005). De las sustancias químicas registradas que presentan efectos toxicológicos conocidos, 350 son cancerígenas (Naciones Unidas, 2015).
La investigación del cáncer ocupacional ha sido poco abordada, a pesar de que su descubrimiento fue hace más de dos siglos cuando Percivall Pott por los años de 1775, mediante una serie de observaciones, demostró la existencia de cáncer de escroto en unos operarios que tenían como oficio la limpieza de chimeneas en Londres, enfermedad ocasionada por la exposición a agentes ambientales procedentes de su labor (Arroyave E, 2010). El riesgo de exposición a agentes químicos se puede presentar en diferentes áreas de una empresa, es decir, no solo está asociado a la manipulación directa de las sustancias, sino en cualquier otro sector del ambiente de trabajo (Cabrera y Álvarez, 2018). La principal vía de exposición se da a través de la inhalación, debido a la volatilidad de las sustancias, puesto que sus partículas se pueden dispersar en el aire con mayor facilidad, depositándose en las vías respiratorias. Consecutivamente, también puede 
haber riesgo dérmico o por ingestión asociado a su naturaleza, lo que demanda tomar medidas preventivas en cuanto a su manipulación (Kim. M y Sang. H 2018; UNIÓN SINDICAL DE MADRID REGIÓN DE CC.OO, 2006). Según la Organización Internacional del Trabajo (OIT) a nivel mundial se calcula que, de 2 millones de muertes laborales que ocurren cada año en el mundo, 440.000 se producen como resultado de la exposición a sustancias químicas (PNUMA, 2007). En el 2003, la Unión Europea reportó que se producen anualmente 32.000 muertes por cáncer, 16.000 enfermedades cutáneas, 6.700 enfermedades respiratorias, 500 enfermedades oculares y 570 enfermedades del sistema nervioso central (Sánchez y Peláez, 2014)

El Consejo Colombiano de Seguridad CISPROQUIM, informó que en el primer semestre del 2011 se recibieron 4492 reportes de emergencia, con un aumento del $13 \%$ con respecto al mismo periodo del año 2010, en el que se reportaron 3974 casos (CISPROQUIM, 2012). El Ministerio de la Protección Social de Colombia a través del Instituto Nacional de Cancerología, ha desarrollado desde el año 2013 el proyecto "Sistema de Vigilancia Epidemiológica del Cáncer Ocupacional" (SIVECAO) debido al problema de salud pública a nivel mundial de cáncer ocupacional (Ministerio del Trabajo, 2014; Gil, Gamboa y Orejuela, M, 2015 ). Según datos reportados por el fondo colombiano de enfermedades la Cuenta de Alto Costo (CAC), en Colombia hubo 275.348 personas diagnosticadas con cáncer durante el periodo analizado (2 de enero de 2017 al 1 de enero del 2018). Durante este periodo se reportaron 37.630 casos nuevos y 19.814 personas con diagnóstico de cáncer fallecieron. El cáncer de mama, de próstata, y los tumores en la piel fueron los más frecuentes entre la población atendida en el sistema de salud de Colombia en el mismo periodo (Ministerio de salud, 2018).

El presente estudio se realizó en una planta procesadora de alimentos cárnicos del departamento del Cauca (Colombia). Aunque las principales actividades desarrolladas en la organización no están relacionadas directamente con procesos químicos en los que se involucre el uso de sustancias peligrosas, requieren el uso de una amplia variedad de estas, que se encuentran en los diferentes almacenes dentro de la empresa, lo cual hace que parte de sus empleados se encuentren expuestos tanto directa como indirectamente. Por lo tanto, el objetivo del estudio fue identificar y clasificar las sustancias químicas $y$, de este modo, poder analizar los niveles de riesgo de algunos agentes cancerígenos que se emplean en actividades diarias en la empresa.

\section{MATERIALES Y MÉTODOS}

El estudio se desarrolló en una planta de derivados cárnicos, mediante diferentes fases que incluyen: (1) descripción de las características generales de la población; (2) diagnostico mediante la descripción cualitativa de las sustancias químicas peligrosas con especial atención en las sustancias cancerígenas; y (3) análisis de la información recolectada

Para la primera fase, se realizó una búsqueda bibliográfica en bases de datos especializadas como Pubmed, Scopus, proquest, Scielo, Google scholar y páginas web institucionales como la International Agency for Research on Cancer (IARC)y la Organización Mundial de la 
Salud (OMS) (Figura 1), así como reportes de entes gubernamentales. También se hizo una descripción de las características generales de la población, se tomó como referencia la base de datos suministrada por la empresa tanto del inventario como del personal expuesto a riesgo químico en cada una de las áreas laborales, en este punto se consideró importante el tiempo y cantidad de sustancia a la que se encuentra en exposición.

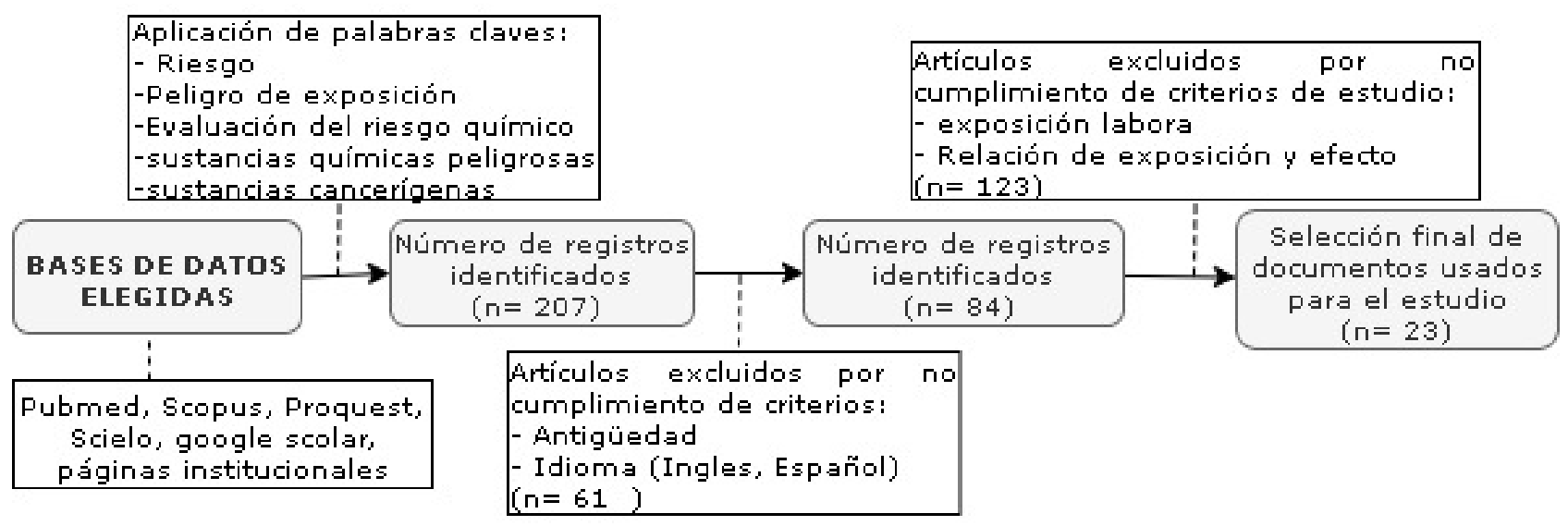

Figura 1. Resumen de búsqueda y selección de literatura utilizada en el estudio.

Fuente: Autores

La fase de diagnóstico se realizó mediante una serie de visitas a los diferentes almacenes en la planta, donde se logró la actualización del inventario, la identificación y clasificación del tipo de producto químico, las condiciones de infraestructura de las zonas del almacén, el estado de los envases de las sustancias químicas, las correspondientes fichas de seguridad, además se inspeccionaron los procedimientos de trabajo que practican mediante listas de chequeo.

Para el análisis de la información obtenida se tuvo en cuenta las características de estudio como: bandas de peligro, rangos y exposiciones potenciales, fichas guías para las estrategias de control, mediante la aplicación de los fundamentos de la evaluación cualitativa por el método COSHH ESSENTIALS (Instituto Nacional de Seguridad e Higiene en el Trabajo (INSHT), 2017; Guananga. A, 2019; Herrick, 1998)

\section{RESULTADOS Y DISCUSIÓN}

Descripción de las características generales de la población: en la planta procesadora de alimentos cárnicos se encuentra una población trabajadora de 228 personas en la totalidad de sus áreas. La empresa cuenta con 20 almacenes donde se acopian los diferentes productos. La revisión del inventario de cada uno de los almacenes y el chequeo de la información encontrada en la ficha de datos de seguridad permitió determinar que en siete de los almacenes hay de sustancias químicas clasificadas como peligrosas (Tabla 1 ). 
Tabla 1. Sitios de almacenamiento de riesgo potencial.

\begin{tabular}{|c|c|c|c|}
\hline $\mathbf{N}^{\circ}$ & Almacenes & PE* & Clasificación \\
\hline 1 & Almacén General de Lubricantes & \multirow{5}{*}{2} & \multirow{4}{*}{ Primaria } \\
\hline 2 & Almacén de Lubricantes Simout & & \\
\hline 3 & Almacén de Obras Civiles Pinturas MasterPlac & & \\
\hline 4 & Almacén de Obras Civiles Morteros MasterPlac & & \\
\hline 5 & Herramientero Mantenimiento & & \multirow{3}{*}{ Secundaria } \\
\hline 6 & Centro de Análisis & 3 & \\
\hline 7 & Cuarto Ondina PTAR & 2 & \\
\hline
\end{tabular}

* Personas expuestas

Fuente: Autores.

Como se muestra en la Tabla 1 , se determinó que los almacenes generales de lubricantes, lubricantes Simout y obras civiles pinturas Masterpac son los lugares con mayor capacidad de abastecimiento de sustancias químicas y corresponden a una clasificación primaria. Dentro de la clasificación secundaria, con menor contenido de productos químicos, se hallan los almacenes de herramientero-mantenimiento, centro de análisis y cuarto ondina Planta de tratamiento de aguas residuales (PTAR). Encontrándose siete personas con exposición directa y constante al manipular o supervisar las áreas de almacenes.

Diagnostico mediante la descripción cualitativa de las sustancias químicas cancerígenas: se identificaron 407 sustancias químicas (Figura 2 ), de las cuales solo un $6 \%$ no representan clasificación peligrosa y el $91 \%$ son potencialmente peligrosas, según su composición físico-química, propiedades toxicológicas y efectos específicos sobre la salud humana.

Se evidenció que un 3\% (11 sustancias) de los productos que se manejan en actividades diarias se consideran cancerígenos y altamente tóxicos. Los riesgos para la salud de quienes están expuestos a este tipo de sustancias en su trabajo es un tema que cada día cobra más importancia porque, en la actividad diaria, al inhalar, manipular o estar cerca de sustancias químicas durante su vida laboral, se pueden inducir en el trabajador alteraciones en la vía aérea dependiendo de la concentración, manipulación, exposición, susceptibilidad del trabajador. Estos agentes químicos pueden producir una diversidad de efectos irritantes alérgicos, tóxicos e incluso cancerígenos (Fierro Valle, 2019; Pratt, 2002).

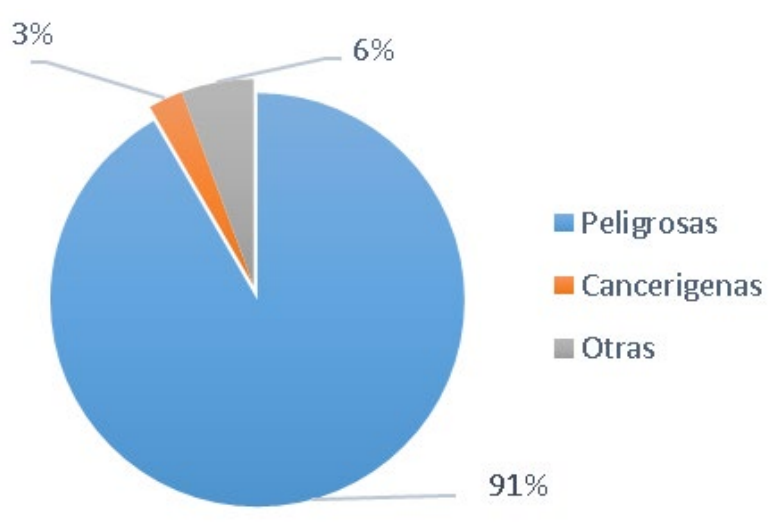

Figura 2. Caracterización de sustancias químicas.

Fuente: Autores.

En la tabla 2, se hace un listado de las 11 sustancias cancerígenas y los hallazgos más 
representativos, como lo es su estado de la materia, la cantidad que se maneja por mes y clasificación de potencial cancerígeno, según la clasificación de la categoría del Sistema Globalmente Armonizado (SGA) (Decreto N 1496 de 2018). La sustancia con mayor uso y exposición es el cemento Portland, con una cantidad de rotación aproximada de $400 \mathrm{~kg}$ por mes, que además esta categorizada como $1 \mathrm{~A}$, es decir, un producto altamente peligroso debido a su composición porque puede contener trazas de sílice cristalina respirable y cromo hexavalente, que están clasificados como carcinógenos para los seres humanos por las Notas Técnicas de Prevención (NTP) y el Centro Internacional de Investigaciones sobre el Cáncer (IARC)
(Domingo-Pueyo et al., 2014, International Agency for research on Cancer, 2012).

El Cemento portland puede generar polvo respirable durante su manejo; sin embargo, una sobreexposición podría causar neumoconiosis, una enfermedad respiratoria causada por inhalación de este mineral, o silicosis, una enfermedad respiratoria generada por inhalación de polvo de sílice, situaciones que conllevan a la inflamación y fibrosis del tejido pulmonar (Álvarez, A. et. al 2017; Rahmani et. al 2018). La exposición a polvo respirable se debe monitorear y controlar para evitar daños pulmonares en trabajadores expuestos (Domingo-Pueyo et al, 2014; Argos, 2012).

Tabla 2. Sustancias químicas cancerígenas ( ${ }^{1} \mathbf{1 A}$ : Cancerígeno para humanos

2: Probablemente cancerígeno para humanos).

\begin{tabular}{|c|c|c|c|}
\hline Nombre comercial & Estado & Categoria $^{1}$ & Cantidad/mes \\
\hline Agar YGC Agar & Sólido & 2 & $8 \mathrm{~kg}$ \\
\hline Cemento Portland & Sólido & $1 \mathrm{~A}$ & $400 \mathrm{~kg}$ \\
\hline Diclorometano & Líquido & 2 & $1 \mathrm{~L}$ \\
\hline DQO Solución B & Líquido & $1 \mathrm{~A}$ & $0.5 \mathrm{~L}$ \\
\hline Gasolina & Líquido & 2 & $7.6 \mathrm{~L}$ \\
\hline Loctite 243 & Líquido & 2 & $0.05 \mathrm{~L}$ \\
\hline Loctite 515 & Líquido & 2 & $0.05 \mathrm{~L}$ \\
\hline Sikadur 32 Primer Comp A & Sólido & 2 & $2 \mathrm{~kg}$ \\
\hline Sikadur 32 Primer Comp B & Sólido & 2 & $2 \mathrm{~kg}$ \\
\hline Sikadur Panel Comp A & Sólido & 2 & $2 \mathrm{~kg}$ \\
\hline Sikadur Panel Comp B & Sólido & 2 & $2 \mathrm{~kg}$ \\
\hline
\end{tabular}

Fuente: Autores

Se realizó una amplia revisión de literatura sobre la gestión de riesgo químico y, se recolectó información del inventario y de inspecciones en las diferentes áreas de almacenamiento de la planta. Después de este proceso, se aplicaron los formatos para identificación del riesgo, a través de la lista de chequeo de seguridad, en la utilización de productos químicos basada en la evaluación cualitativa por el método COSHH ESSENTIALS (Control of Substances Hazardous to Health). Esta es una metodología de evaluación de riesgos para identificar la medida de control adecuada, que ayuda a las empresas a cumplir las normativa sobre control de sustancias (Instituto Nacional de Seguridad e Higiene en el Trabajo (INSHT), 2017; 
Guananga, 2019; Herrick, 1998). En esta etapa se tuvieron en cuenta variables de evaluación del riesgo; condiciones de utilización y medios técnicos de control, envasado y etiquetado de los productos químicos; plan de emergencia; y la vigilancia de la salud, formación e información a los trabajadores.

Tratamiento y análisis de la información: se realizó un análisis cualitativo de las sustancias químicas cancerígenas utilizadas en la empresa mediante la metodología simplificada del riesgo químico de la "caja de herramientas de control químico internacional" orientada por la Organización Internacional del Trabajo, que es una guía específica para establecer un esquema de protección en el lugar de trabajo (International Labour Organization (ILO), 2017).

De esta manera se considera la exposición a sustancias químicas peligrosas por vía inhalatoria y vía dérmica. En el desarrollo del estudio se hizo especial énfasis en el manejo del cemento Portland por las cantidades que se manejan, el personal expuesto y la falta de control en los elementos de protección personal. Por este motivo, las herramientas para la gestión del riesgo químico (Instituto Nacional de Seguridad e Higiene en el Trabajo (INSHT), 2017), consideran factores relacionados con la exposición por ambas vías, y establecen un nivel de riesgo que se asocia a una estrategia de control para su uso (Figura 3). Estos factores forman el corazón del método de "control banding" desarrollado por HSE (Health and Safety Executive) y COSHH Essentials. Para el análisis de los datos se realizó un estudio cualitativo en cuatro etapas necesarias para evaluar la volatilidad de las sustancias que representan mayor riesgo en la salud de los trabajadores. En este estudio cualitativo se tienen en cuenta los factores mencionados en la Figura 3.

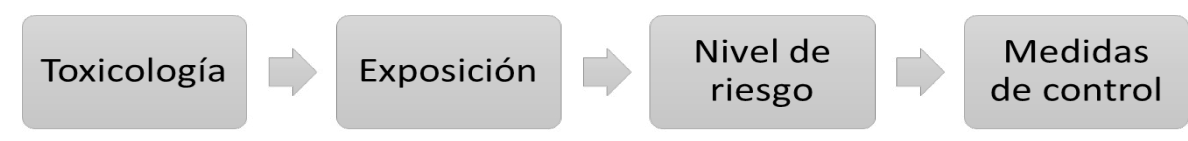

Figura 3. Factores considerados en el procedimiento de evaluación de riesgo químico.

Fuente: Autores.

- Etapa 1: peligro para la salud.

En esta etapa se realizó una clasificación de las sustancias químicas de interés según su afectación a la salud, los métodos cualitativos de evaluación de riesgos, parten de los criterios toxicológicos y por tanto de los efectos para la salud, para establecer los distintos niveles o bandas de peligro, el método COS$\mathrm{HH}$ Essentials establece cinco bandas: A, B, C, D y E. Cada una de las cinco bandas (AE) representa un intervalo de concentración de distribución logarítmica, que se alcanzaría cuando se emplean los métodos de control adecuados y por tanto será el rango objetivo. 
Por ejemplo: la banda de peligro $\mathrm{B}$ representa un rango objetivo de 0,1 a $1 \mathrm{mg} / \mathrm{m}^{3}$ para polvo, que sería el rango de exposición si se empleara un sistema de extracción localizada, banda de control o abordaje 2. Así, las frases de riesgo (Frases $\mathrm{R}$ ) se distribuyen dentro de esas cinco bandas o intervalos de concentración. Asignación de Frases $\mathrm{R}$ a las bandas o grupos de peligro). (Instituto nacional de seguridad e Higiene en el trabajo, 2017. p 31). De acuerdo a lo estipulado por las frases de riesgo para las sustancias químicas mediante el uso de las tablas de correspondencias se presenta la tabla de clasificación de riesgo para las 11 sustancias químicas estudiadas, se tuvo en cuenta la clasificación de dichas sustancias mediante sus etiquetas y fichas de datos de seguridad.

Tabla 3. Clasificación en grupos de riesgo según frases $R$

\begin{tabular}{c|l|c} 
No & Sustancia & Grupo de riesgo* \\
\hline 1 & Agar YGC Agar & D \\
\hline 2 & Cemento Portland & C, E \& S \\
\hline 3 & Diclorometano & C, D, E \& S \\
\hline 4 & DQO Solución B & B, E \& S \\
\hline 5 & Gasolina & D\&S \\
\hline 6 & Loctite 243 & D \& S \\
\hline 7 & Loctite 515 & D \& S \\
\hline 8 & Sikadur 32 Primer Comp A & D, E \& S \\
\hline 9 & Sikadur 32 Primer Comp B & D \& S \\
\hline 10 & Sikadur Panel Comp A & C, D, E \& S \\
\hline 11 & Sikadur Panel Comp B & \\
\hline
\end{tabular}

*(A: $1-10 \mathrm{mg} / \mathrm{m} 3$ polvo;50-500 ppm vapor B: 0,1-1 mg/m3 polvo; 5-50 ppm vapor C: 0,01-0.1 mg/m3 polvo; 0,5-5 ppm vapor D: 0,01 mg/m3 polvo; 0,5 ppm vapor E: Buscar asesoramiento experto S: Prevención o reducción de la exposición dérmica y ocular (Instituto Nacional de Seguridad e Higiene en el Trabajo, 2017. p 33).

Fuente: Autores.

- Etapa 2: exposición potencial.

En esta etapa se realizó la distribución de sustancias según la cantidad usada y la volatilidad o pulverulencia. En la cantidad utilizada, se determina la practicidad y facilidad mediante niveles muy alto, alto, medio, bajo, evidenciadas en la Tabla 4. En la segunda banda se encontraron los rangos de exposición y exposiciones potenciales, mediante el uso de propiedades físico-químicas (volatilidad y pulverulencia), como se muestra en la Tabla 5. 
Tabla 4. Clasificación escala de uso.

\begin{tabular}{|c|c|c|c|}
\hline Sustancia & Estado & Cantidad & Escala de uso* \\
\hline Agar YGC Agar & Sólido & $\mathrm{kg}$ & Medio \\
\hline Cemento Portland & Sólido & $\mathrm{kg}$ & Medio \\
\hline Diclorometano & Líquido & $\mathrm{mL}$ & Bajo \\
\hline DQO Solución B & Líquido & $\mathrm{mL}$ & Bajo \\
\hline Gasolina & Líquido & L & Medio \\
\hline Loctite 243 & Líquido & $\mathrm{mL}$ & Bajo \\
\hline Loctite 515 & Líquido & $\mathrm{mL}$ & Bajo \\
\hline Sikadur 32 Primer Comp A & Sólido & $\mathrm{kg}$ & Medio \\
\hline Sikadur 32 Primer Comp B & Sólido & $\mathrm{kg}$ & Medio \\
\hline Sikadur Panel Comp A & Sólido & $\mathrm{kg}$ & Medio \\
\hline Sikadur Panel Comp B & Sólido & $\mathrm{kg}$ & Majo \\
\hline
\end{tabular}

* Muy Alto:4 Alto:3 Medio:2 Bajo:1 (Instituto Nacional de Seguridad e Higiene en el Trabajo , 2017).

Fuente: Autores

Tabla 5. Clasificación de volatilidad.

\begin{tabular}{|c|c|c|c|}
\hline Sustancia & Estado & $* \mathbf{C C P}$ & *Volatilidad \\
\hline Agar YGC Agar & Sólido & Baja & \\
\hline Cemento Portland & Sólido & Aalta & \\
\hline Diclorometano & Líquido & & Alta \\
\hline DQO Solución B & Líquido & & Baja \\
\hline Gasolina & Líquido & & Media \\
\hline Loctite 243 & Líquido & & Baja \\
\hline Loctite 515 & Líquido & & Baja \\
\hline Sikadur 32 Primer Comp A & Sólido & Baja & \\
\hline Sikadur 32 Primer Comp B & Sólido & Baja & \\
\hline Sikadur Panel Comp A & Sólido & Baja & \\
\hline Sikadur Panel Comp B & Sólido & Baja & \\
\hline
\end{tabular}

* CPP:Capacidad para convertirse en polvo; Volatiliada (Muy alta: 1-10 mg/m³ polvo;50-500 ppm vapor, Alta: 0,1-1 $\mathrm{mg} / \mathrm{m}^{3}$ polvo; 5-50 ppm vapor Media: 0,01-0.1 $\mathrm{mg} / \mathrm{m}^{3}$ polvo; 0,5-5 ppm vapor Baja: 0,01 $\mathrm{mg} / \mathrm{m}^{3} \mathrm{polvo;} 0,5 \mathrm{ppm}$ vapor) (Instituto Nacional de Seguridad e Higiene en el Trabajo , 2017. p 33).

Fuente: Autores.

- Etapa 3: Evaluación de riesgos genérica. Para la etapa de evaluación de riesgo se tuvo en cuenta la combinación de factores de peligro para la salud y exposición potencial que determinan el nivel de control deseado. Se determinaron cuatro niveles (Abordaje 1, 
Abordaje 2, Abordaje 3, Abordaje 4) en función de los parámetros de toxicidad, exposición, nivel de riesgo como se muestra en las tablas 3, 4 y 5, siendo el 4 una consideración especial, ya que es el punto más crítico y de mayor peligrosidad. En la Tabla 6 se muestra que las sustancias de mayor atención son el cemento portland, DQO solución B, Gasolina, Sikadur 32 Primer Comp B, y Sikadur Panel Comp B.

Tabla 6. Nivel de control específico.

\begin{tabular}{l|l} 
Sustancia & Nivel de control \\
\hline Agar YGC Agar & Abordaje 3 \\
\hline Cemento Portland & Abordaje 3, Abordaje 4. \\
\hline Diclorometano & Abordaje 1, Abordaje 3. \\
\hline DQO Solución B & Abordaje 1, Abordaje 2, Abordaje 4. \\
\hline Gasolina & Abordaje 2, Abordaje 4. \\
\hline Loctite 243 & Abordaje 2. \\
\hline Loctite 515 & Abordaje 2. \\
\hline Sikadur 32 Primer Comp A & Abordaje 3. \\
\hline Sikadur 32 Primer Comp B & Abordaje 3, Abordaje 4. \\
\hline Sikadur Panel Comp A & Abordaje 3. \\
\hline Sikadur Panel Comp B & Abordaje 2, Abordaje 3, Abordaje 4. \\
\hline \multicolumn{2}{c}{ Abl factor de riesgo está dado por el nivel de deficiencia y presenta la siguiente descripción: } \\
(Instituto Nacional de Seguridad e Higiene en el Trabajo, 2017).
\end{tabular}

Fuente: Autores

- Etapa 4. Medida de control.

En esta etapa se tomaron las sustancias que representaba un alto grado de peligrosidad (Abordaje 4). En este tipo de enfoque de control se busca conseguir el control adecuado, en cuanto a su utilización. Mediante fichas guía para las estrategias de control, asesoramiento directo con ejemplos prácticos, procedimientos de operación, actividades y pautas de actuación. Descrito en hojas de control, así como se muestra en la Tabla 7.

Tabla 7. Hojas de control de tarea

\begin{tabular}{l|l}
$\begin{array}{l}\text { Sustancia } \\
\text { Cemento Portland }\end{array}$ & $\begin{array}{l}\text { Hoja de control de la tarea (Instituto Nacional } \\
\text { de Seguridad e Higiene en el Trabajo (INSHT), 2017) }\end{array}$ \\
\hline DQO Solución B & Hoja 3, Hoja 4. \\
\hline Gasolina & Hoja 1, Hoja 2, Hoja 4. \\
\hline Sikadur 32 Primer Comp B & Hoja 2, Hoja 4. \\
\hline Sikadur Panel Comp B & Hoja 3, Hoja 4. \\
\hline
\end{tabular}


Según el número de abordaje, las sustancias que poseen un alto rango de riesgo químico y volatilidad son: Cemento Portland, DQO Solución y Gasolina. Estas sustancias tienen un nivel de deficiencia numero 4 (muy alto), que corresponde a un control especial. Se debe optar por seguir medidas específicas y obtener orientación de personal experto, considerando que son sutancias que pueden dispersarse con facilidad en el aire y causar efectos muy graves para la salud, debido a los periodos de contacto, y desencadenar en toxicidad multiple, debido a que su abordaje de peligro tiene clasificación muy alta. Sikadur 32 Primer Comp B y Sikadur Panel Comp B son sustancias que presentan un rango medio del nivel de riesgo cancerígeno. Las demás sustancias poseen de igual manera un riesgo cancerígeno, pero no tienen alta capacidad para dispersarse en el ambiente, por lo que las personas más afectadas son las que tienen contacto directo con el producto.

\section{CONCLUSIONES}

Mediante el diagnóstico de inventario, se identificaron 407 sustancias en la planta procesadora de alimentos cárnicos, de las que el $91 \%$ se clasifican como sustancias químicas peligrosas; un $6 \%$ no presentan riesgo a la salud; y el $3 \%$ restante corresponde a sustancias químicas con potencial efecto cancerígeno. Esas sustancias son el cemento portland, DQO solución B, Gasolina, Sikadur 32 Primer Comp B, Sikadur Panel Comp B, determinadas por la evaluación de riesgo como nivel de abordaje 4, siendo considerado de carácter especial por ser el punto más crítico y de mayor peligrosidad.

El estudio desarrollado sirve de punto de partida en el análisis de la situación actual de los riesgos que pueden sufrir los empleados de la empresa y cuál es el sector más críti$\mathrm{CO}$, De donde se pueden desprender futuras investigaciones y controles de vigilancia, que proporcionen información válida y confiable para el diseño de intervenciones para el control del cáncer relacionados a enfermedades laborales asociada a factores como el tiempo de exposición y la concentración.

Comentarios: se adiciona material suplementario donde se relacionan algunas tablas complementarias tomadas de INSHT (2017), Así como ejemplo de hojas de control para el cemento portland, según especificaciones Instituto Nacional de Seguridad e Higiene en el Trabajo (INSHT), 2017.

\section{CONTRIBUCIÓN DE LA AUTORÍA}

Primer autor (Ángela Arias): metodología, investigación, análisis de datos, conceptualización, escritura - borrador original. Segundo Autor: (Efren Ramos): escritura - revisión y edición Tercer autor (Zuly Delgado): investigación, conceptualización, análisis de datos, escritura - revisión y edición.

\section{LITERATURA CITADA}

Álvarez, A., Zambrano, V., Mazzini, M., y Zambrano, W. (2017). Mucosa nasal y determinación de alteraciones, citopatológicas por exposición al cemento. Dominio de las Ciencias, 3(4), 602-612.

Argos. (2012). "Portland Cement HOJA DE DATOS DE SEGURIDAD.":9. Argos. https://www.argos. co/Media/USA/Page/sds/Argos-Portland-Cement-Safety-Data-Sheets-Spanish.pdf

Arroyave E, A. (2010). serie de observaciones, demostró la existencia de cáncer de escroto en unos operarios que tenían como oficio la limpieza de chimeneas en Londres. (Tesina de master). Universidad Politécnica de Valencia. Valencia

Cabrera, J., y Alvarez X. (2018). Identificación de Riesgos Laborales En Los Talleres de Metales, Maderas, Cerámicas y Aula de Pintura En La Facultad de Artes de La Universidad de Cuenca. Revista de La Facultad de Ciencias Químicas. Cuenca. p. 1-6.

Calera, R., y Alfonso, A. (2005). Riesgo Químico Laboral: Elementos Para Un Diagnóstico En España. Revista Española de Salud Pública. 79(2): 283-95. 
CISPROQUIM, Consejo Colombiano de Seguridad. (2012). GUÍAS AMBIENTALES DE ALMACENAMIENTO Y TRANSPORTE. p 167.

Domingo-Pueyo, A., Valero, S., y Wanden-Berghe, C. (2014). EFFECTS OF OCCUPATIONAL EXPOSURE TO CHROMIUM AND ITS COMPOUNDS: A SYSTEMATIC REVIEW. Archivos de Prevencion de Riesgos Laborales 17(3): 142-53. Doi: https:// doi.org/10.12961/aprl.2014.17.3.03

Fierro Valle, L. P. (2019). Enfermedades respiratorias y factores de riesgo por exposición a sustancias químicas en los empleados de la empresa Industrias Químicas ASPROQUIN LTDA durante el segundo semestre del 2018 (Tesis de especialización). Universidad de Manizales.

Gil, M., Gamboa, O., y Orjuela, M. E. (2015). Antecedentes ocupacionales documentados en la Historia clínica de pacientes con diagnóstico de cáncer pulmonar. Revista Colombiana de Cancerología, 19(3), 156-165.

Guananga, A (2019). Evaluación Higiénica Cualitativa Del Riesgo Químico Por Exposición a Sustancias Químicas Peligrosas En Un Laboratorio de Análisis Químico Ambiental (Tesis de maestría). Universidad de Cuenca. Cuenca.

Herrick, R. (1998). "HIGIENE INDUSTRIAL." en Enciclopedia de salud y seguridad, Madrid, pp 30-32

Instituto Nacional de Seguridad e Higiene en el Trabajo (INSHT). (2017). HERRAMIENTAS PARA LA GESTIÓN DEL RIESGO QUÍMICO. Barcelona: Instituto Nacional de Seguridad e Higiene en el Trabajo.

https://www.insst.es/documents/94886/96076/ Herramientas+para+la+gestion+del+riesg o+quimico.pdf/ca44ff68-bde2-4b96-af67$1477 f 9 f 0 b f 76 ? t=1527065689115$

International Agency for research on Cancer. (2012). A Review of Human Carcinogens. Biological Agents, 100 B, p. 485.

International Labour Organization (ILO). (2017). "International Chemical Control Toolkit.", Identification of the skin and eye contact hazards (Group S). https://www.ilo.org/legacy/english/protection/safework/ctrl_banding/toolkit/icct/guide.pdf

Kim, M., y Hoon S. (2018). Evaluation of a Chemical Risk Assessment Method of South Korea for
Chemicals Classified as Carcinogenic, Mutagenic or Reprotoxic (CMR). International Journal of Occupational Medicine and Environmental Health, 31 (4), pp 491-501. https://doi.org/10.13075/ijomeh.1896.01125.

Ministerio de Salud. (2018). Fondo Colombiano de enfermedades de alto costo. Situación del cáncer en la población adulta atendida en el SGSSS de Colombia. 1-290.

Ministerio del Trabajo. (2014). Instituto Nacional de Cancerología (ESE). Sistema de Vigilancia Epidemiológica Del Cáncer Ocupacional' (SIVECAO).": 116.

Naciones Unidas. (2015). SISTEMA GLOBALMENTE ARMONIZADO DE CLASIFICACIÓN Y ETIQUETADO DE PRODUCTOS QUÍMICOS (SGA), 578.

Pratt, I. (2002). Global Harmonisation of Classification and Labelling of Hazardous Chemicals. Toxicology Letters, 128(1-3), 5-15. Recuperado de http://linkinghub.elsevier.com/retrieve/pii/ S037842740100529X (22 de marzo de 2019).

PNUMA, Programa de Naciones Unidas para el Medio Ambiente, (2007). Enfoque Estratégico Para La Gestión de Productos Químicos a Nivel Internacional. Ginebra.

Rahmani, A., Almatroudi, A., Babiker, A., Khan. A., y Alsahli, M. (2018). Effect of Exposure to Cement Dust among the Workers: An Evaluation of Health Related Complications. Open access Macedonian journal of medical sciences, 6(6), pp 1159-1162.

Sánchez, F., y Peláez, J. (2014). Eficacia de Las Medidas Preventivas y Evaluación Del Riesgo Químico En Una Empresa Avícola. Revista Colombiana de Salud Ocupacional, 4(2), 5-11.

Sistema globalmente Armonizado de Clasificación y etiquetado de productos químicos (SGA) (2013), Anexo 1 tablas Resumen de la Clasificación y etiquetado, Quinta Ed, Revisada, Naciones Unidas, Nueva York y Ginebra, P,269-286. https://unece. org/fileadmin/DAM/trans/danger/publi/ghs/ghs_ rev05/Spanish/ST-SG-AC10-30-Rev5sp.pdf

UNIÓN SINDICAL DE MADRID REGIÓN DE CC.OO. (2006). Exposición Laboral a Productos Químicos En La Comunidad de Madrid. Madrid. 


\section{(c) (i) (2) (2)

